\title{
Efficient Modified FCS Algorithm for Compressed MR Image Reconstruction
}

\author{
S.Jagadeesh $^{1}$, Dr.E.Nagabhooshanam ${ }^{2}$, N.Esther Diana Nithya ${ }^{3}$ \\ ${ }^{I}$ (Assoc. Prof. \& Head of the Department, Electronics and Communications Engineering Department, SSJ \\ Engineering College/ JNTU, Hyderabad, Andhra Pradesh, India) \\ ${ }^{2}$ (Dr. Prof. \& Head of the Department, Electronics and Communications Engineering Department, Sridevi \\ Women's Engineering College/ JNTU, Hyderabad, Andhra Pradesh, India) \\ ${ }^{3}$ (Electronics and Communications Engineering Department, SSJ Engineering College/ JNTU, Hyderabad, \\ Andhra Pradesh, India)
}

\begin{abstract}
In recent work, FCSA has shown an efficient algorithm for MR image reconstruction by linear combination of least square data fitting, TV and L1 norm regularization algorithms. FCSA based reconstructed MR image had improved results compared to CS methods. In our paper, we are going to propose a reconstruction algorithm which improves the FCSA algorithm in two algorithms. Firstly, MR image will be enhanced by proposed bicubic and bilateral method. Secondly, the enhanced MR image will be resolved by proposed image resolution method. These two algorithms improve the compression technique and image reconstruction. Our performance evaluation results have shown better results comparatively.
\end{abstract}

Keywords: Bicubic and bilinear method, FCSA, least square data fitting, L1 norm regularization, TV.

\section{INTRODUCTION}

Compressed Sensing has become extensive applications in medical image processing $[1,2]$. Compressive sensing is a non-adaptive signal sampling framework that collects samples from sparse signals; which can be used to reconstruct the original signal. Magnetic Resonance Imaging is technique to reduce image acquisition time. Compressed Sensing is a recent development in image processing with technical elements that allow an image to be compressed, and recovered which reduce the number of samples during image acquisition.

In Medical Image analysis, medical data from patient reports are taken in the form of DICOM images. These DICOM images will provide the information about the patient depending on the disease or replacement of organ. In diagnostics device applications patient data has to be computerized in to digital formats. These digital formatted DICOM images has to be analyzed for exact patient data.

Huge amount of DICOM images are stored in diagnostic device, where the patient scan copies are stored in digital form. These data may be varied based on the patient's information to be analyzed. The amount of data storage in diagnostic machine may vary, mostly on patient's data, format of the scan segments and type of diagnostics taking place. For these diagnostic machines, they require large memory location to store the patient's data in DICOM format. But diagnostic machines are made with a fixed memory space, further improvement of these are not possible.

And in real time application medical images have to be transmitted based on the availability of patient location, for this we require an efficient method to transmit the patients data without any loss. While transmitting patient information in the form of DICOM images, they have to be converted in to a standard image format.

To transmit DICOM image, without the loss of patient's information, we need to implement an efficient algorithm which improves the computational complexity of the algorithm developed.

Apart from these, to store large amount of patient's information we need an image compression technique which will improve the storage capacity of the patient's information in diagnostic machines. MR image compression includes two stages. Firstly, image of given parameters will be compressed based on compressive sensing and secondly compressed image have to be reconstructed efficiently by improving reconstruction accuracy, iteration time, peak signal to noise ratio (PSNR) and compression ratio (CR). As a forward step, there are proposed compressive techniques for MR image compression. They are

1. Iterative Shrinkage Thresholding Algorithm (ISTA)

2. Fast Iterative Shrinkage Thresholding Algorithm (FISTA)

3. Composite Splitting Denoising (CSD)

4. Composite Splitting Algorithms (CSA)

5. Fast Composite Splitting Algorithms (FCSA)

6. Fast Composite Splitting Algorithms (FCSA-MRI) 
These compressive techniques have shown great results in compressing the MR image, we are proposing an enhanced and improved FCSA algorithms which improves the MR image reconstruction. We had studied practically and implemented the proposed algorithm on the data collected from three patients scan DICOM images. Patient's data which we had taken for analysis are tabulated in table 1.

\begin{tabular}{|l|l|l|l|}
\hline & \multicolumn{1}{|c|}{ Patient 01 } & Patient 02 & Patient 03 \\
\hline Scan MR image & & \\
& & & \\
& & & \\
& & & \\
\hline Part of MR image & 3DMR_Renal_Arteries & 3DMR_Heart & 3DMR_Spine \\
\hline Size of MR image & 10.9KB & 19 KB & 106 KB \\
\hline
\end{tabular}

We had taken these patients MR image and analyzed these images with previously proposed methods and compared with our proposed algorithm of modified FCSA algorithm.

Our proposed algorithm with comparative results, iteration time and PSNR had shown better results than the proposed algorithms. In our proposed method we are using bicubic and bilateral methods with super resolution method to improve the compression rate of 20: 1 value and reconstruction accuracy of $81 \%$ with PSNR of 38.73 which shows a reliable transmission of MR image through a communication link in the vicinity of patient's location. Based on our proposed algorithm, MR image can be encoded using a MR image encoder which provides a loss less reconstruction of the original image.

Our proposed algorithm had shown better results compared to previously proposed algorithms.

\section{Methodology: Algorithms ReVieW}

Reviews of algorithms [1,2]; developed to improve the compression ratio and recovery time of original compressive sensing image.

Algorithm 1: Iterative Shrinkage Thresholding Algorithm (ISTA): Reconstructed image is expressed by equation:

$$
\mathrm{x}^{\mathrm{k}}=\operatorname{prox}_{\rho}(\mathrm{g})\left(\mathrm{x}^{\mathrm{k}-1}-\rho \boxminus \mathrm{f}\left(\mathrm{x}^{\mathrm{k}-1}\right)\right) \ldots \text { Eqn.1 }
$$

Algorithm 2: Fast Iterative Shrinkage Thresholding Algorithm (FISTA)

$$
\begin{gathered}
\mathrm{x}^{\mathrm{k}}=\operatorname{prox}_{\rho}(\mathrm{g})\left(\mathrm{r}^{\mathrm{k}}-\rho \oplus f\left(\mathrm{x}^{\mathrm{k}}\right)\right) \\
\mathrm{t}^{\mathrm{k}+1}=\left(1+\sqrt{\left.\left(1+4\left(\mathrm{t}^{\mathrm{k}}\right) 2\right)\right) / 2}\right. \\
\mathrm{r}^{\mathrm{k}+1}=\mathrm{x}^{\mathrm{k}}+\left(\mathrm{t}^{\mathrm{k}}-1 / \mathrm{t}^{\mathrm{k}+1}\right)\left(\mathrm{x}^{\mathrm{k}}-\mathrm{x}^{\mathrm{k}-1}\right) \ldots \text { Eqn.2 }
\end{gathered}
$$

Algorithm 3: Composite Splitting Denoising (CSD)

$$
x^{i}=\arg \min _{x}(1 / 2 m \rho)\left\|x-z_{i}^{j-1}\right\|^{2}+g_{i}\left(B_{i} x\right) \ldots \text { Eqn.3 }
$$

Algorithm 4: Composite Splitting Algorithms (CSA)

$$
\mathrm{y}_{\mathrm{i}}^{\mathrm{k}}=\operatorname{prox}_{\rho}\left(\mathrm{g}_{\mathrm{i}}\right)\left(\mathrm{B}_{\mathrm{i}}\left(\mathrm{x}^{\mathrm{k}-1}-\left((1 / \mathrm{L}) \mathrm{Ðf}_{\mathrm{i}}\left(\mathrm{x}^{\mathrm{k}-1}\right)\right)\right)\right) \ldots \text { Eqn.4 }
$$

Algorithm 5: Fast Composite Splitting Algorithms (FCSA)

$$
\mathrm{y}_{\mathrm{i}}^{\mathrm{k}}=\operatorname{prox}_{\rho}\left(\mathrm{g}_{\mathrm{i}}\right)\left(\mathrm{B}_{\mathrm{i}}\left(\mathrm{x}^{\mathrm{k}}-\left((1 / \mathrm{L}) \mathrm{Ðf}_{\mathrm{i}}\left(\mathrm{x}^{\mathrm{k}}\right)\right)\right)\right) \ldots \text {... Eqn.5 }
$$

Algorithm 6: Fast Composite Splitting Algorithms -MRI

$$
\begin{aligned}
& \mathrm{x}_{\mathrm{g}}=\mathrm{r}^{\mathrm{k}}-\rho \oplus f\left(\mathrm{r}^{\mathrm{k}}\right) \\
& \mathrm{x}_{1}=\operatorname{prox}_{\rho}\left(2 \alpha\|\mathrm{x}\|_{\mathrm{TV}}\right)\left(\mathrm{x}_{\mathrm{g}}\right) \\
& \mathrm{x}_{2}=\operatorname{prox} \rho\left(2 \beta\|\Phi \mathrm{x}\|_{1}\right)\left(\mathrm{x}_{\mathrm{g}}\right) \\
& \mathrm{xk}=\left(\mathrm{x}_{1}+\mathrm{x}_{2}\right) / 2 \\
& \mathrm{t}^{\mathrm{k}+1}=\left(1+\sqrt{ }\left(1+4\left(\mathrm{t}^{\mathrm{k}}\right) 2\right)\right) / 2 \\
& \mathrm{r}^{\mathrm{k}+1}=\mathrm{x}^{\mathrm{k}}+\left(\mathrm{t}^{\mathrm{k}}-1 / \mathrm{t}^{\mathrm{k}+1}\right)\left(\mathrm{x}^{\mathrm{k}}-\mathrm{x}^{\mathrm{k}-1}\right) \ldots \text {..Eqn.6 }
\end{aligned}
$$

Algorithm 7: Our proposed Modified Fast Composite Splitting Algorithm -MRI 


$$
\begin{gathered}
\mathrm{x}_{\mathrm{g}}=\mathrm{r}^{\mathrm{k}+1}-\rho \oplus \mathrm{f}\left(\mathrm{r}^{\mathrm{k}+1}\right) \\
\mathrm{x}_{1}=\operatorname{prox}_{\mathrm{\rho}}\left(2 \alpha\|\mathrm{x}\|_{\mathrm{TV}}+2.5\|\partial\|_{\mathrm{TV}}\right)\left(\mathrm{x}_{\mathrm{g}}+\mathrm{x}_{\mathrm{i}}\right) \\
\mathrm{x}_{2}=\operatorname{prox} \rho\left(2 \beta\|\Phi \mathrm{x}\|_{1}+2.5\|\hat{\omega}\|_{1}\right)\left(\mathrm{x}_{\mathrm{g}}+\mathrm{x}_{\mathrm{i}}\right) \\
\mathrm{xk}=\left(\left(\mathrm{x}_{1}+\mathrm{x}_{2}\right) / 2\right)+\left(\|\partial\|_{\mathrm{TV}} *\|\hat{\omega}\|_{1}\right) \\
\mathrm{t}^{\mathrm{k}+1}=\left(1+\sqrt{ }\left(1+\left(\mathrm{t}^{\mathrm{k}}\right)\right)\right) / 2 \\
\mathrm{r}^{\mathrm{k}+1}=\mathrm{x}^{\mathrm{k}}+\left(\mathrm{t}^{\mathrm{k}}-1 / \mathrm{t}^{\mathrm{k}+1}\right)\left(\mathrm{x}^{\mathrm{k}}-\mathrm{x}^{\mathrm{k}-1}\right) \ldots \text { Eqn.7 }
\end{gathered}
$$

In our paper, we are going to propose a MR Image reconstruction algorithm which improves the FCSA algorithm in two algorithms based on high resolution image enhancement methods [6, 7] improving the compression technique comparatively.

This paper is organized as follows. In chapter 3, proposed algorithm has been illustrated with a MR image containing patient scanned kidneys image. In chapter 4 comparative results have been shown with the previous proposed methods. Chapter 5 clearly illustrates the conclusion of the research algorithm proposed. Future research work has been proposed in chapter 6.

\section{Progressive ApProach To OUR Proposed CoMpression Model Modified Fast COMPOSITE SPLITTING ALGORITHM (MFCSA)}

In our propose algorithm of MR image compressive sensing algorithm, an improved version of FCSA is presented. Proposed algorithm is as follows:

Step 01: Select a MR image for analysis.

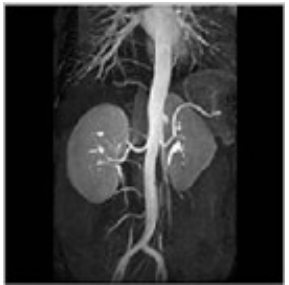

Figure 1: Original MR Image.

Step 02: Resize the image in to [256 256]

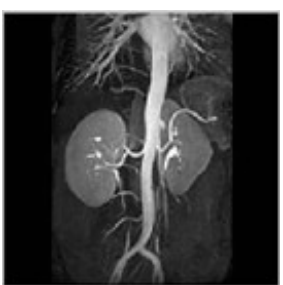

Figure 2: Resized MR Image.

Step 03: Let the MR image be represented by Z.

Step 04: Analyze the partial FT $\mathrm{x}$ and sampling parameter $\mathrm{y}$, as $\mathrm{y}=\mathrm{Zx}$ [1].

Step 05: Enhance the MR image by bicubic and bilateral method using

a. Assign $w=5$; sigma $=[2$ 2.5] and [2.5 2];

b. Compute sigma_ $r=100 *$ sigma(2);

c. Design filter coefficients $(-w: w,-w: w)$;

d. Compute signal filter values $G=\exp \left(-\left(X .^{\wedge} 2+Y .^{\wedge} 2\right) /\left(2 * \operatorname{sigma}(1)^{\wedge} 2\right)\right)$;

e. Apply bilateral filter.

f. Extract local regions iMin, iMax, jMin and jMax. by computing JJ=A(iMin:iMax,jMin:jMax);

g. Compute Gaussian intensity weights by $H=\exp (-(J J-A(i, j)))^{\wedge} 2 /\left(2 *\right.$ sigma $\left.\left.(2)^{\wedge} 2\right)\right)$;

h. Calculate bilateral filter response by $F=H .{ }^{*} G((i M i n: i M a x)-i+w+1,(j M i n: j M a x)-j+w+1)$;

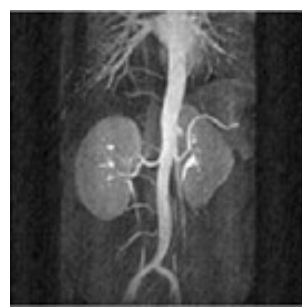

Figure 3: Enhanced MR image by bicubic and bilateral method. 
Step 06: Further image resolution method is applied on enhanced MR image super interpolation resolution method using
a. Compute Sparse on given image
b. Assign lambda $=.1 ; k=0 ;$ tol $=.001$;
c. Compute interpolation method by resolution technique

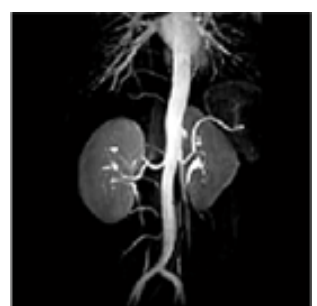

Figure 4: Enhanced MR image by super interpolation resolution method.

Step 07: High resolution and enhanced MR image will be further processed with modified FCSA algorithm using
a. Assign iterations $=0$; iterno $=$ iterno +1 ;
b. Assign $y p=y ; y g=y-\left(A^{\prime *}\left(A^{*} y\right)-A t b\right) / L x$;
c. Compute denoising TV on iterative shrinking image.
d. Compute $x_{g}=r^{k+1}-\rho \boxminus f\left(r^{k+1}\right)$
e. Compute $x_{1}=\operatorname{prox}_{\rho}\left(2 \alpha\|x\|_{T V}+2.5\|\partial\|_{T V}\right)\left(x_{g}+x_{i}\right)$
f. Compute $x_{2}=\operatorname{proxp}\left(2 \beta\|\Phi x\|_{I}+2.5\|\mid \dot{\omega}\|_{I}\right)\left(x_{g}+x_{i}\right)$
g. Compute $x k=\left(\left(x_{1}+x_{2}\right) / 2\right)+\left(\|\partial\|_{T V} *\|\hat{\omega}\|_{1}\right)$
h. Compute $t^{k+1}=\left(1+\sqrt{\left.\left(1+\left(t^{k}\right)\right)\right) / 2}\right.$
i. $\quad$ Compute $r^{k+1}=x^{k}+\left(t^{k}-1 / t^{k+1}\right)\left(x^{k}-x^{k-1}\right)$

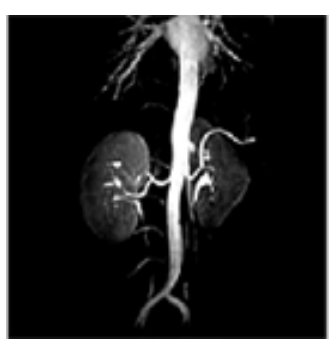

Figure 5: Enhanced MR image by modified FCSA algorithm.

Step 08: Proposed modified FCSA compressed MR image will be analyzed with PSNR values of original MR image.

Step 09: Reconstructed MR image will be compared with FCSA, CSA, RecPF, TVCMRI and Sparse MRI algorithms $[3,4]$ with iteration time and PSNR.

Step 10: Comparative Results are analyzed with Modified FCSA Algorithm, High Resolution FCSA Algorithm and High Resolution with Interpolation FCSA Algorithm. These results have shown a better improvement in the FCSA Algorithm. Comparative results for 3DMR_Renal_Arteries_MR Image are tabulated in Table 1.

Table 1 Comparative Results: 3DMR Renal Arteries

\begin{tabular}{|c|c|c|c|c|c|c|c|c|c|c|c|}
\hline \multicolumn{4}{|c|}{ Modified FCSA } & \multicolumn{4}{|c|}{ HRFCSA } & \multicolumn{4}{|c|}{$\begin{array}{l}\text { HRIFCSA } \\
\end{array}$} \\
\hline $\begin{array}{c}\text { METH } \\
\text { OD }\end{array}$ & IMAGE & $\begin{array}{c}\text { ITER_TI } \\
\text { ME }\end{array}$ & $\begin{array}{c}\text { PSN } \\
\text { R } \\
\end{array}$ & $\begin{array}{c}\text { METH } \\
\text { OD }\end{array}$ & IMAGE & $\begin{array}{c}\text { ITER_T } \\
\text { IME }\end{array}$ & $\begin{array}{l}\text { PPS } \\
\text { NR } \\
\end{array}$ & $\begin{array}{c}\text { METH } \\
\text { OD }\end{array}$ & IMAGE & $\begin{array}{c}\text { ITER_TI } \\
\text { ME }\end{array}$ & $\begin{array}{c}\text { PS } \\
\text { NR } \\
\end{array}$ \\
\hline & Original & & & CG & & $24.85 \mathrm{sec}$ & 9.74 & CG & & $24.87 \mathrm{sec}$ & $\begin{array}{c}8.8 \\
8\end{array}$ \\
\hline CG & & $25.29 \mathrm{sec}$ & $\begin{array}{c}11.7 \\
2\end{array}$ & $\begin{array}{c}\text { TVCM } \\
\text { RI }\end{array}$ & & $5.19 \mathrm{sec}$ & 14.16 & $\begin{array}{c}\text { TVCM } \\
\text { RI }\end{array}$ & & $5.21 \mathrm{sec}$ & $\begin{array}{l}13 . \\
65\end{array}$ \\
\hline
\end{tabular}




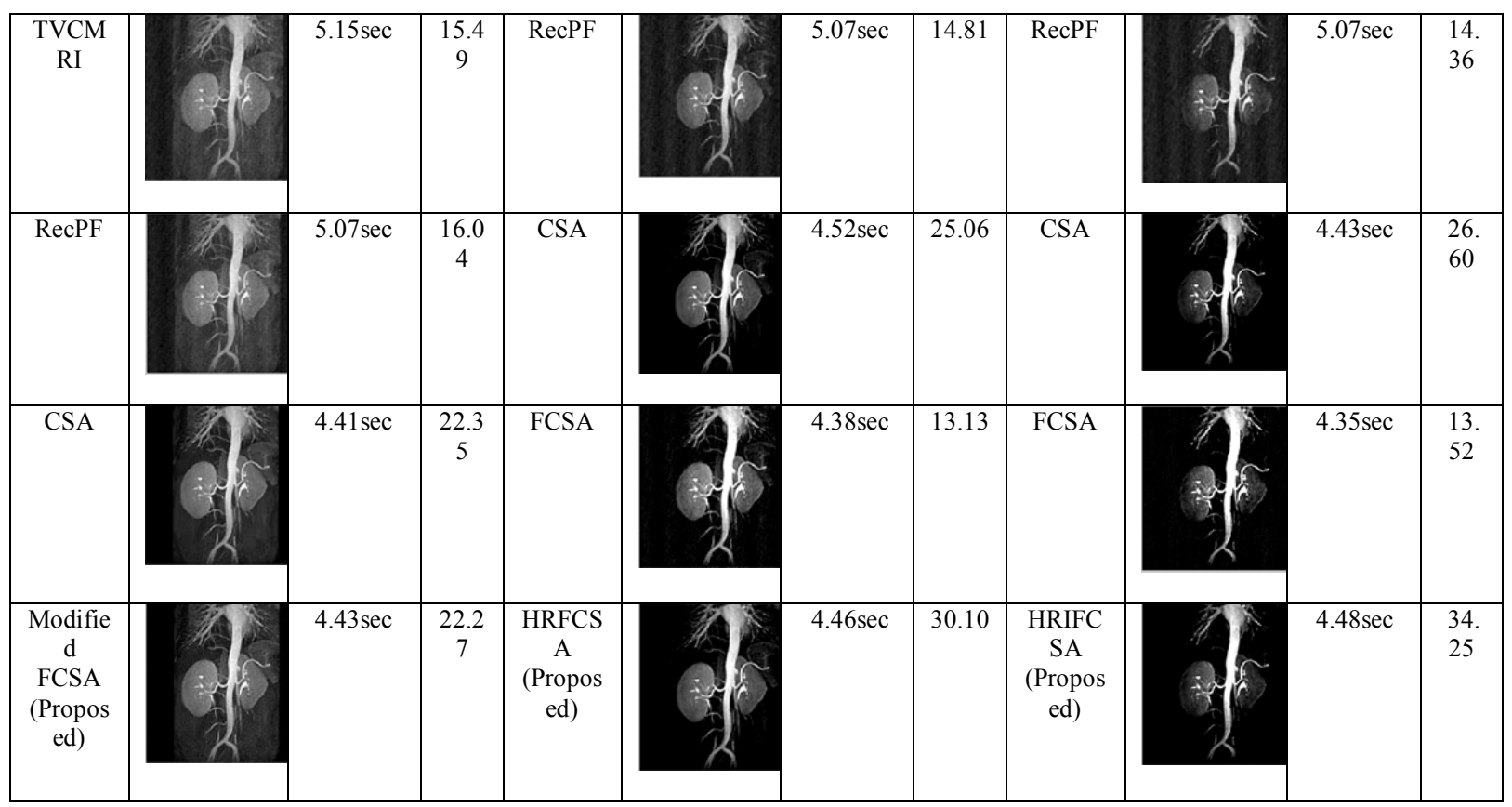

\section{Performance Evaluation And Comparative Results}

In our paper, we had taken three MR images Renal Arteries, Heart and Spine. These MR images are analyzed with proposed FCSA algorithm and previously proposed methods. Comparative results are tabulated in Table 1,2 and 3.

Table 2 Comparative Results: Heart

\begin{tabular}{|c|c|c|c|c|c|c|c|c|c|c|c|}
\hline \multicolumn{4}{|c|}{ Modified FCSA } & \multicolumn{4}{|c|}{ HRFCSA } & \multicolumn{4}{|c|}{ HRIFCSA } \\
\hline $\begin{array}{l}\text { METHO } \\
\text { D }\end{array}$ & IMAGE & $\begin{array}{l}\text { ITER_TI } \\
\text { ME }\end{array}$ & $\begin{array}{l}\text { PSN } \\
\text { R }\end{array}$ & $\begin{array}{l}\text { METHO } \\
\text { D }\end{array}$ & IMAGE & $\begin{array}{l}\text { ITER_TI } \\
\text { ME }\end{array}$ & $\begin{array}{l}\text { PSN } \\
\text { R }\end{array}$ & $\begin{array}{l}\text { METHO } \\
\text { D }\end{array}$ & IMAGE & $\begin{array}{l}\text { ITER_TI } \\
\text { ME }\end{array}$ & $\begin{array}{l}\text { PSN } \\
\text { R }\end{array}$ \\
\hline & Original & & & CG & & $25.69 \mathrm{sec}$ & 7.03 & CG & & $25.01 \mathrm{sec}$ & 6.31 \\
\hline CG & & $26.04 \mathrm{sec}$ & 9.86 & $\begin{array}{l}\text { TVCMR } \\
\text { I }\end{array}$ & & $5.18 \mathrm{sec}$ & $\begin{array}{l}12.9 \\
1\end{array}$ & $\begin{array}{l}\text { TVCMR } \\
\text { I }\end{array}$ & & $5.21 \mathrm{sec}$ & $\begin{array}{l}12.1 \\
7\end{array}$ \\
\hline $\begin{array}{l}\text { TVCMR } \\
\text { I }\end{array}$ & & $5.26 \mathrm{sec}$ & $\begin{array}{l}14.4 \\
3\end{array}$ & RecPF & & $5.05 \mathrm{sec}$ & $\begin{array}{l}13.9 \\
7\end{array}$ & RecPF & & $5.13 \mathrm{sec}$ & $\begin{array}{l}13.5 \\
0\end{array}$ \\
\hline RecPF & & $5.07 \mathrm{sec}$ & $\begin{array}{l}15.2 \\
0\end{array}$ & CSA & & $4.45 \mathrm{sec}$ & $\begin{array}{l}26.2 \\
4\end{array}$ & $\mathrm{CSA}$ & & $4.45 \mathrm{sec}$ & $\begin{array}{l}29.2 \\
2\end{array}$ \\
\hline CSA & & $4.43 \mathrm{sec}$ & $\begin{array}{l}18.3 \\
2\end{array}$ & FCSA & & $4.43 \mathrm{sec}$ & $\begin{array}{l}13.7 \\
2\end{array}$ & FCSA & & $4.37 \mathrm{sec}$ & $\begin{array}{l}15.1 \\
2\end{array}$ \\
\hline
\end{tabular}




\begin{tabular}{|c|c|c|c|c|c|c|c|c|}
\hline $\begin{array}{l}\text { Modifie } \\
\text { d FCSA } \\
\text { (Propose } \\
\text { d) }\end{array}$ & $4.63 \mathrm{sec}$ & $\begin{array}{l}16.4 \\
6\end{array}$ & $\begin{array}{l}\text { HRFCS } \\
\text { A } \\
\text { (Propose } \\
\text { d) }\end{array}$ & $4.46 \mathrm{sec}$ & $\begin{array}{l}34.4 \\
4\end{array}$ & $\begin{array}{l}\text { HRIFCS } \\
\text { A } \\
\text { (Propose } \\
\text { d) }\end{array}$ & $4.48 \mathrm{sec}$ & $\begin{array}{l}38.7 \\
3\end{array}$ \\
\hline
\end{tabular}

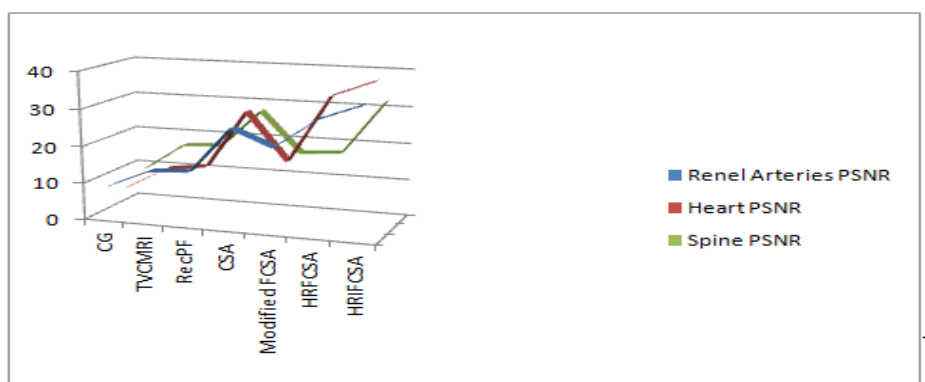

Figure 6: Comparative Results between CG, TVCMRI, RecPF, CSA, Modified FCSA, HRFCSA and HRIFCSA for Arteries, Heart and Spine MR Images.

Table 3 Comparative Results: Spine

\begin{tabular}{|c|c|c|c|c|c|c|c|c|c|c|c|}
\hline \multicolumn{4}{|c|}{ Modified FCSA } & \multicolumn{4}{|c|}{ HRFCSA } & \multicolumn{4}{|c|}{ HRIFCSA } \\
\hline $\begin{array}{l}\text { METH } \\
\text { OD }\end{array}$ & IMAGE & $\begin{array}{c}\text { ITER_TI } \\
\text { ME }\end{array}$ & $\begin{array}{c}\text { PSN } \\
\text { R }\end{array}$ & $\begin{array}{l}\text { METH } \\
\text { OD }\end{array}$ & IMAGE & $\begin{array}{c}\text { ITER_TI } \\
\text { ME }\end{array}$ & PSNR & $\begin{array}{l}\text { METH } \\
\text { OD }\end{array}$ & IMAGE & $\begin{array}{c}\text { ITER_TI } \\
\text { ME }\end{array}$ & PSNR \\
\hline & Original & & & CG & & $24.82 \mathrm{sec}$ & $\begin{array}{l}11.5738 \\
32\end{array}$ & $\mathrm{CG}$ & & $24.84 \mathrm{sec}$ & $\begin{array}{l}9.4151 \\
03\end{array}$ \\
\hline $\mathrm{CG}$ & & $25.10 \mathrm{sec}$ & $\begin{array}{l}11.3 \\
8\end{array}$ & $\begin{array}{l}\text { TVCM } \\
\text { RI }\end{array}$ & & $5.23 \mathrm{sec}$ & 17.17 & $\begin{array}{l}\text { TVCM } \\
\text { RI }\end{array}$ & & $5.23 \mathrm{sec}$ & 16.68 \\
\hline $\begin{array}{l}\text { TVCM } \\
\text { RI }\end{array}$ & & $5.30 \mathrm{sec}$ & $\begin{array}{l}16.6 \\
2\end{array}$ & RecPF & & $5.01 \mathrm{sec}$ & 17.79 & RecPF & & $5.02 \mathrm{sec}$ & 17.34 \\
\hline RecPF & & $5.05 \mathrm{sec}$ & $\begin{array}{l}17.3 \\
4\end{array}$ & CSA & & $4.46 \mathrm{sec}$ & 19.80 & $\mathrm{CSA}$ & & $4.45 \mathrm{sec}$ & 27.63 \\
\hline CSA & & $4.48 \mathrm{sec}$ & 19.2 & FCSA & & $4.41 \mathrm{sec}$ & 10.42 & FCSA & & $4.41 \mathrm{sec}$ & 13.70 \\
\hline $\begin{array}{l}\text { Modifie } \\
\text { d FCSA } \\
\text { (Propos } \\
\text { ed) }\end{array}$ & & $4.52 \mathrm{sec}$ & $\begin{array}{l}16.7 \\
3\end{array}$ & $\begin{array}{l}\text { HRFCS } \\
\text { A } \\
\text { (Propos } \\
\text { ed) }\end{array}$ & & $4.48 \mathrm{sec}$ & 17.51 & $\begin{array}{l}\text { HRIFC } \\
\text { SA } \\
\text { (Propos } \\
\text { ed) }\end{array}$ & & $4.45 \mathrm{sec}$ & 31.85 \\
\hline
\end{tabular}

Comparative results shown improved FCSA compared to the FCSA algorithm. So from the comparative results iteration time and PSNR clearly indicates that the high resolution and interpolation method will improve FCSA Algorithm. 


\section{CONCLUSION}

The proposed modified FCSA, HRFCSA and HRIFCSA algorithm can be applicable to low resolution MR images and for volumetric analysis of MR images. Our proposed algorithm had shown better comparative results. An efficient compression technique for MR Images is proposed in our algorithm.

\section{FUTURE RESEARCH}

Our proposed method can be further applicable to CT images and DICOM Images where special extraction of image is necessary. Further our algorithm can be applicable to high compressive image decoder for compressive sensing.

\section{REFERENCES}

[1] S. Chen, D. Donoho, and M. Saunders. Atomic decomposition by basis pursuit. Statistical and Scientific Computing, pages 33-61, 1999.

[2] I. F. Claerbout and F. Muir. Robust modeling with erratic data. Geophysics Magazine, 38(5):826-844, October 1973.

[3] P. L. Combettes and V. R. Wajs. Signal recovery by proximal forward-backward splitting. Multiscale Modeling and Simulation, 4(4):1168-1200, 2005.

[4] G. Cormode, M. Datar, P. Indyk, and S. Muthukrishnan. Comparing data streams using hamming norms (how to zero in). In VLDB, pages 335-345, 2002.

[5] C.R.Vogel. Non-convergence of the 1-curve regularization parameter selection method. Inverse Problems, 12:535-547, 1996.

[6] I. Daubechies. Orthonormal bases for compactly supported wavelets. Communications on Pure and Applied Mathematics, pages 909-996, 1988.

[7] Daubechies. Ten Lectures on Wavelets. SIAM Press, Philadelphia, PA, 1992. 